\title{
WEAK-POLYNOMIAL CONVERGENCE ON A BANACH SPACE
}

\author{
J. A. JARAMILLO AND A. PRIETO
}

(Communicated by Theodore W. Gamelin)

\begin{abstract}
We show that any super-reflexive Banach space is a $\Lambda$-space (i.e., the weak-polynomial convergence for sequences implies the norm convergence). We introduce the notion of $\kappa$-space (i.e., a Banach space where the weakpolynomial convergence for sequences is different from the weak convergence) and we prove that if a dual Banach space $Z$ is a $\kappa$-space with the approximation property, then the uniform algebra $A(B)$ on the unit ball of $Z$ generated by the weak-star continuous polynomials is not tight.
\end{abstract}

We shall be concerned in this note with some questions, posed by Carne, Cole, and Gamelin in [3], involving the weak-polynomial convergence and its relation to the tightness of certain algebras of analytic functions on a Banach space.

Let $X$ be a (real or complex) Banach space. For $m=1,2, \ldots$ let $\mathscr{P}\left({ }^{m} X\right)$ denote the Banach space of all continuous $m$-homogeneous polynomials on $X$, endowed with its usual norm; that is, each $P \in \mathscr{P}\left({ }^{m} X\right)$ is a map of the form $P(x)=A\left(x, \ldots,,^{m)} x\right)$, where $A$ is a continuous, $m$-linear (scalar-valued) form on $X \times \ldots m) \times X$.

Also, let $\mathscr{P}(X)$ denote the space of all continuous polynomials on $X$; that is, each $P \in \mathscr{P}(X)$ is a finite sum $P=P_{0}+P_{1}+\cdots+P_{n}$, where $P_{0}$ is constant and each $P_{m} \in \mathscr{P}\left({ }^{m} X\right)$ for $m=1,2, \ldots, n$.

In [3] a sequence $\left(x_{j}\right) \subset X$ is said to be weak-polynomial convergent to $x \in X$ if $P\left(x_{j}\right) \rightarrow P(x)$ for all $P \in \mathscr{P}(X)$; and the space $X$ is defined to be a $\Lambda$-space, if whenever $\left(x_{j}\right)$ is a sequence in $X$ that is weak-polynomial convergent to 0 , then $\left\|x_{j}\right\| \rightarrow 0$.

It is shown in [3] that $l_{p}$ is a $\Lambda$-space for $1 \leq p<\infty$; it is also shown that $L_{p}(\mu)$ is a $\Lambda$-space for $2 \leq p<\infty$ and $L_{1}[0,1]$ is not a $\Lambda$-space, and the question is posed as to whether $L_{p}(\mu)$ is a $\Lambda$-space for $1<p<2$. Our next result will provide an affirmative answer to this question.

First we recall that super-reflexive Banach spaces can be defined as those spaces that admit an equivalent uniformly convex norm. In particular, spaces $L_{p}(\mu)$ are super-reflexive for $1<p<\infty$ and any measure $\mu$ (see, e.g., [8, Chapter 3]).

We shall use the following fact: If $X$ is super-reflexive, then there exists

Received by the editors June 7, 1991 and, in revised form, September 23, 1991.

1991 Mathematics Subject Classification. Primary 46J15; Secondary 46B10.

Both authors were partially supported by DGICYT PB 87-1031.

(C) 1993 American Mathematical Society $0002-9939 / 93 \$ 1.00+\$ .25$ per page 
some $p \quad(1<p<\infty)$ such that each bounded sequence $\left(x_{j}\right)$ in $X$ has a weakly $p$-convergent subsequence $\left(x_{j_{k}}\right)$; that is, there exists $x \in X$ so that $\sum_{k=1}^{\infty}\left|x^{*}\left(x_{j_{k}}-x\right)\right|^{p}<+\infty$ for all $x^{*} \in X^{*}$. This is proved in [5, Proposition 3.1] using a suitable characterization of super-reflexivity given by James [12].

Theorem 1. Every super-reflexive Banach space is a $\Lambda$-space.

Proof. Let $\left(x_{j}\right)$ be a sequence in a super-reflexive space $X$ such that $P\left(x_{j}\right) \rightarrow$ $P(0)$ for all $P \in \mathscr{P}(X)$, but $\left\|x_{j}\right\| \nrightarrow 0$. There exist some $\varepsilon>0$ and a subsequence, which we still denote $\left(x_{j}\right)$ such that $\left\|x_{j}\right\| \geq \varepsilon$; by the BessagaPełczynski selection principle, $\left(x_{j}\right)$ can be considered a basic sequence in $X$ (see, e.g., [8]). Let $Y$ denote the closed subspace of $X$ spanned by $\left(x_{j}\right)$, and let $\left(x_{j}^{*}\right)$ be the corresponding sequence of functional coefficients associated to $\left(x_{j}\right)$. Then $\left(x_{j}^{*}\right)$ is a bounded sequence in $Y^{*}$. Each $x_{j}^{*}$ can be extended to a functional $\hat{x}_{j}^{*} \in X^{*}$ with the same norm; therefore, $\left(\hat{x}_{j}^{*}\right)$ is a bounded sequence in $X^{*}$. $X^{*}$, however, is super-reflexive since $X$ is (see, e.g., [16]) and then, as we remarked before, it follows from [5] that there exist some $p(1<p<\infty)$, some $x^{*} \in X^{*}$, and a subsequence $\left(\hat{x}_{j_{k}}^{*}\right)$ such that

$$
\sum_{k=1}^{\infty}\left|\left(\hat{x}_{j_{k}}^{*}-x^{*}\right)(x)\right|^{p}<+\infty \quad \text { for all } x \in X .
$$

Let $N \in \mathbb{N}$ with $N \geq p$. Then $\sum_{k=1}^{\infty}\left|\left(\hat{x}_{j_{k}}^{*}-x^{*}\right)(x)\right|^{N}<+\infty$ for all $x \in X$ and hence, by the Banach-Steinhaus type theorem for homogeneous polynomials (see, e.g., $[6,4.17])$ the expression

$$
P(x)=\sum_{k=1}^{\infty}\left(\left(\hat{x}_{j_{k}}^{*}-x^{*}\right)(x)\right)^{N}, \quad x \in X,
$$

defines a continuous $N$-homogeneous polynomial on $X$. Now for each $y \in Y$ we have $y=\sum_{j=1}^{\infty} x_{j}^{*}(y) x_{j}$ and, therefore, $x_{j}^{*}(y) \rightarrow 0$. Since $\left(\hat{x}_{j_{k}}^{*}-x^{*}\right)(x) \rightarrow 0$ for each $x \in X$, it follows that $x^{*}(y)=0$ for all $y \in Y$. In particular, we obtain that $P\left(x_{j_{k}}\right)=1$ for all $k$, and this contradicts the hypothesis on the polynomial convergence of $\left(x_{j}\right)$.

Remark. In [5] a Banach space $X$ is defined to be in the class $W_{p} \quad(1<p<\infty)$ when each bounded sequence in $X$ admits a weakly $p$-convergent subsequence. The proof of Theorem 1 shows that if $X^{*}$ is in the class $W_{p}$ for some $p$ $(1<p<\infty)$ then $X$ is a $\Lambda$-space. In particular, it follows from [5] that the dual Tsirelson space $T$ and the space $\left(\bigoplus l_{\infty}^{n}\right)_{l_{p}}(1<p<\infty)$ are $\Lambda$-spaces, since the original Tsirelson space $T^{*}$ is in $W_{p}$ for all $p(1<p<\infty)$ and $\left(\left(\bigoplus l_{\infty}^{n}\right) l_{p}\right)^{*}=\left(\bigoplus l_{1}^{n}\right) l_{p^{*}}$ is in $W_{p}$.

The authors thank Jesús F. Castillo for providing this remark (and other useful comments).

The notion of $\Lambda$-space was introduced in [3] in relation to the tightness of certain algebras of analytic functions on a (complex) Banach space. We recall that a uniform algebra $A$ on a compact space $K$ is said to be tight on $K$ if, for all $g \in C(K)$, the Hankel-type operator $S_{g}: A \rightarrow C(K) / A$ defined by $S_{g}(f)=f g+A$ is weakly compact.

Now let $Z$ be a complex dual Banach space with open unit ball $B$, and let $A(B)$ be the algebra generated by the weak *-continuous linear functionals on 
the closed unit ball $\bar{B}$ (regarded as functions on the weak*-compact set $\bar{B}$ ). It is proved in [3] that if $A(B)$ is tight on $\bar{B}$ then $Z$ is reflexive. Therefore, we shall be mainly concerned with reflexive Banach spaces. It is also proved that if $Z$ is an infinite-dimensional $\Lambda$-space with the metric approximation property, then $A(B)$ is not tight. We will obtain an extension of this last result for reflexive $Z$.

First we define a Banach space $X$ to be a $\kappa$-space if there exists a weakly null sequence in $X$ that is not weak-polynomial convergent to 0 . In other words, $X$ is a $\kappa$-space if and only if there exists a continuous polynomial $P$ on $X$ that is not weakly sequentially continuous; it is clear that $P$ can be chosen to be $m$-homogeneous for some $m$.

The following results show that many classes of reflexive Banach spaces are $\kappa$-spaces.

Proposition 1. Let $X$ be a reflexive, infinite-dimensional $\Lambda$-space. Then $X$ is a $\kappa$-space.

Proof. Since $X$ is an infinite-dimensional reflexive space, by the JosefsonNissenzweig theorem (see, e.g., [9, Chapter 12]) there exists a weakly null sequence $\left(x_{j}\right)$ in $X$ that is not norm-convergent. Since $X$ is $\Lambda$-space, $\left(x_{j}\right)$ is not weak-polynomial convergent and, therefore, $X$ is a $\kappa$-space.

Proposition 2. If $X$ is a reflexive Banach space and a quotient $Y$ of $X$ is a $\kappa$-space, then $X$ is a $\kappa$-space.

Proof. Let $T: X \rightarrow Y$ be continuous, linear onto $Y$. Choose a sequence $\left(y_{j}\right)$ in $Y$ with $y_{j} \rightarrow 0$ weakly and $P \in \mathscr{P}\left({ }^{m} Y\right)$ with $\left|P\left(y_{j}\right)\right| \geq \varepsilon$ for some $\varepsilon>0$. We can apply the open mapping theorem to find a bounded sequence $\left(x_{j}\right)$ in $X$ with $\left(T x_{j}\right)=\left(y_{j}\right)$. Since $X$ is reflexive, there exist $x \in X$ and a subsequence $\left(x_{j_{k}}\right)$ such that $x_{j_{k}} \rightarrow x$ weakly. Then $y_{j_{k}}=T x_{j_{k}} \rightarrow T x$ weakly and, therefore, $T x=0$. This shows that the polynomial $P \circ T \in \mathscr{P}\left({ }^{m} X\right)$ is not weakly sequentially continuous.

Proposition 3. Suppose that the Banach space $X$ has a weakly null sequence $\left(a_{n}\right)$ verifying that there exists a continuous linear operator $T: X \rightarrow l_{p} \quad(1<p<\infty)$ such that $\left(T a_{n}\right)$ is the canonical basis of $l_{p}$. Then $X$ is a $\kappa$-space.

Proof. Choose $m \in \mathbb{N}$ with $m \geq p$. The series $\sum_{n=1}^{\infty} y_{n}^{m}=P(y)$, with $y=$ $\left(y_{n}\right) \in l_{p}$, defines a continuous $m$-homogeneous polynomial on $l_{p}$. Then $Q=$ $P \circ T \in \mathscr{P}\left({ }^{m} X\right)$ satisfies that $Q\left(a_{n}\right)=1$ and therefore is not weakly sequentially continuous.

Remark. Arguments of this kind have been used in $[2,1]$ to find a continuous polynomial of degree 2 that is not weakly sequentially continuous on the quasireflexive James space $J$ and the dual Tsirelson space $T$, respectively. That is, $J$ and $T$ are $\kappa$-spaces. As remarked before, $T$ is, in fact, a $\Lambda$-space.

We note that Proposition 3 applies whenever $X$ is a Banach space of finite cotype with a weakly null, unconditional basis (see $[14,13])$.

Proposition 4. If a complemented subspace of a Banach space $X$ is a $\kappa$-space, then $X$ is a $\kappa$-space.

Proof. Consider a continuous projection $\Pi$ from $X$ onto $Y$, where the subspace $Y$ is a $\kappa$-space. We can choose a polynomial $P \in \mathscr{P}(Y)$ that is not 
weakly sequentially continuous. Then the polynomial $Q=P \circ \Pi \in \mathscr{P}(X)$ is not weakly sequentially continuous.

This case covers a wide class of operator spaces defined on a $\kappa$-space. For example, the spaces $L(X)$ and $K(X)$, of bounded linear and compact linear operators on $X$, contain a complemented copy of $X$ and, therefore, $L(X)$ and $K(X)$ are $\kappa$-spaces if $X$ is.

An important class of nonreflexive Banach spaces are not $\kappa$-spaces. Recall that the Banach space $X$ has the Dunford-Pettis property if whenever $\left(x_{j}\right) \subset X$ and $\left(\varphi_{j}\right) \subset X^{*}$ are weakly null sequences then $\varphi_{j}\left(x_{j}\right) \rightarrow 0$. Spaces that enjoy the Dunford-Pettis property are $C(K)$ and $L_{1}(\mu)$. Since the restriction of any polynomial on a Dunford-Pettis space to a weakly compact set is weakly continuous (see $[15]$ or $[3,7.1]$ ) it is clear that a space satisfying the DunfordPettis property is not a $\kappa$-space.

The proof of our next result follows the ideas appearing in [3, 9.4].

Theorem 2. Let $Z$ be a complex dual Banach space. Suppose that $Z$ is a $\kappa$-space with the approximation property. Then $A(B)$ is not tight on $\bar{B}$.

Proof. In view of $[3,9.1]$ we can assume that $Z$ is reflexive. Since $Z$ is a $\kappa$-space, for some $m$ there exist a polynomial $P \in \mathscr{P}\left({ }^{m} Z\right)$ and a weakly null sequence $\left(z_{j}\right) \subset \bar{B}$ such that $P\left(z_{j}\right) \nrightarrow P(0)=0$. Suppose that $A(B)$ is tight. Then by $[3,9.1]$ the $A(B)^{* *}$-topology on $\frac{1}{2} \bar{B}$ (considered as a subset of $A(B)^{*}$ ) coincides with the weak topology (as a subset of $Z$ ). Then $F\left(\frac{1}{2} z_{j}\right) \rightarrow F(0)$ for all $F \in A(B)^{* *}$. Now since $Z$ is a dual space with the metric approximation property (see [7, p. 94]), we can apply [3, 3.1 and Theorem 4.4] to obtain that $\left.A(B)^{* *}\right|_{B}=\mathscr{H}^{\infty}(B)$; that is, each bounded holomorphic function on $B$ is the restriction of some element of $A(B)^{* *}$. Therefore $f\left(\frac{1}{2} z_{j}\right) \rightarrow f(0)$ for each $f \in$ $\mathscr{H}^{\infty}(B)$; in particular, $2^{-m} P\left(z_{j}\right)=P\left(\frac{1}{2} z_{j}\right) \rightarrow 0$, which is a contradiction.

Remark. Consider $T^{*}$, the original Tsirelson space; $T^{*}$ is a reflexive space with an unconditional basis, which does not have any quotient isomorphic to $l_{p}$ $(1<p<\infty)$ and which is not a $\kappa$-space (since every continuous polynomial on $T^{*}$ is weakly sequentially continuous; see [1]). Therefore $T^{*}$ is a Banach space for which [3, 9.3 and 9.4] and our Theorem 2 cannot be applied. The tightness of $A(B)$, for $T^{*}$, will be studied below.

Now consider the space $E=T^{*} \times Z$, where $Z$ is a reflexive $\kappa$-space with the approximation property. Since $T^{*}$ is a closed subspace of $E$ and $T^{*}$ is not a $\Lambda$-space, neither is $E$. Nevertheless, $E$ is a reflexive space with the approximation property and $E$ is a $\kappa$-space (because of the same property of $Z$ ). So $E$ provides an example of a Banach space satisfying our Theorem 2, which is not a $\Lambda$-space.

Proposition 5. Let $Z$ be the dual space of a complex separable Banach space. Suppose that there exists a point in the unit sphere $\bar{B} \backslash B$ of $Z$, which is not a complex extreme point. Then $A(B)$ is not tight on $\bar{B}$.

Proof. Since $\bar{B}$ is weak-star metrizable, each point in $\bar{B}$ is a $G_{\delta}$-set. In view of [10, II.12.1] a point in $\bar{B} \backslash B$ is a generalized peak point for $A(B)$ if and only if it is a peak point for $A(B)$. Now choose $z$ in $\bar{B} \backslash B$ that is not a complex extreme point. Therefore, [11, Theorem 4] proves that $z$ is not a peak point for $A(B)$ and, by $[3,9.5], A(B)$ is not tight on $\bar{B}$. 
Proposition 5 provides the arguments showing that $A(B)$ is not tight for the unit ball $B$ of the Tsirelson space $T^{*}$. In fact, it is enough to find $z$ in $\bar{B} \backslash B$ such that $z$ is not a complex extreme point:

Let $\left(e_{j}\right)$ be the canonical unit basis of the dual Tsirelson space $T$ and $\left(e_{j}^{*}\right)$ the associated coefficient functionals of the original Tsirelson space $T^{*}$. By using the analytic description of the norm in $T$ (see [4, I.1]), it is easy to check that $\left\|e_{j}+e_{k}\right\|=1$ if $j \neq k$. Set $z=\frac{1}{2}\left(e_{5}^{*}+e_{6}^{*}\right)$ and $y=\frac{1}{2}\left(e_{7}^{*}+e_{8}^{*}\right)$. For each complex number $\lambda$ with $|\lambda| \leq 1$, consider the vector $z+\lambda y$. Note that the natural projection of $z+\lambda y$ onto the first four coordinates is null, thus $\|2(z+\lambda y)\|=\left\|e_{5}^{*}+e_{6}^{*}+\lambda\left(e_{y}^{*}+e_{8}^{*}\right)\right\| \leq 2$ (see [1, Proposition 5; 4, p. 17]). Since $(z+\lambda y)\left(e_{5}+e_{6}\right)=1$, it is proved that $\|z+\lambda y\|=1$ for each $\lambda$ in the complex unit disc. It follows that $z$ is not an extreme point and $A(B)$ is not tight.

\section{ACKNOWLEDGMENTS}

The authors gratefully acknowledge several helpful conversations with Richard Aron and Miguel Lacruz while this paper was being prepared.

\section{NOTE ADDED IN PROOF}

Using Proposition 2.2 in [R. Aron, C. Hervés, M. Valdivia, Weakly continuous mappings on Banach spaces, J. Funct. Anal. 52 (1983), 189-204], we can equivalently define the notion of $\kappa$-space as follows: A Banach space $X$ is a $\kappa$-space if there exists a weakly Cauchy sequence in $X$ that is not weakpolynomial convergent. Now, applying the Rosenthal-Dor $l_{1}$ theorem [9], we obtain the following sharper version of Proposition 2:

A Banach space not containing $l_{1}$, with a quotient being a $\kappa$-space, is a $\kappa$ space too.

The referee has kindly suggested this point of view.

\section{REFERENCES}

1. R. Alencar, R. Aron, and S. Dineen, A reflexive space of holomorphic functions in infinite many variables, Proc. Amer. Math. Soc. 90 (1984), 407-411.

2. R. Aron and C. Hervés, Weakly sequentially continuous analytic functions on a Banach space, Functional Analysis, Holomorphy and Approximation Theory II (G. Zapata, ed.), North-Holland, Amsterdam, 1984, pp. 23-38.

3. T. Carne, B. Cole, and T. Gamelin, A uniform algebra of analytic functions on a Banach space, Trans. Amer. Math. Soc. 314 (1989), 639-659.

4. P. G. Casazza and T. J. Shura, Tsirelson space, Lecture Notes in Math., vol. 1363, SpringerVerlag, Berlin and New York, 1980.

5. J. F. Castillo and C. Sánchez, Weakly-p-compact, p-Banach-Saks and super-reflexive Banach spaces, preprint.

6. S. B. Chae, Holomorphy and calculus in normed spaces, Marcel Dekker, New York, 1985.

7. M. Day, Normed linear spaces, Springer-Verlag, Berlin and New York, 1973.

8. J. Diestel, Geometry of Banach spaces, Lecture Notes in Math., vol. 485, Springer-Verlag, Berlin and New York, 1975.

9. __ Sequences and series in Banach spaces, Graduate Texts in Math., vol. 92, SpringerVerlag, Berlin and New York, 1984.

10. T. W. Gamelin, Uniform algebras, Chelsea, New York, 1984.

11. J. Globevnik, On interpolation by analytic maps in infinite dimensions, Math. Proc. Cambridge Philos. Soc. 83 (1978), 243-254. 
12. R. James, Super-reflexive spaces with bases, Pacific J. Math. 41 (1972), 409-420.

13. W. Johnson, On finite dimensional subspaces of Banach spaces with local unconditional structure, Studia Math. 51 (1974), 226-240.

14. B. Maurey and G. Pisier, Séries de variables aléatories vectorielles indépendantes et propriétés géométriques des espaces de Banach, Studia Math. 58 (1976), 45-90.

15. R. Ryan, Dunford-Pettis properties, Bull. Polon. Acad. Sci. 27 (1979), 373-379.

16. D. Van Dulst, Reflexive and super-reflexive spaces, Math. Centre Tracts, vol. 102, NorthHolland, Amsterdam, 1982.

Departmento de Analisis Matematico, Universidad Complutense de Madrid, 28040 MADRID, SPAIN

E-mail address, J. A. Jaramillo: JARAMIL@MAT.UCM.ES

E-mail address, A. Prieto: ANGELIN@EMDUCM11.BITNET 